\title{
Power Efficient Hybrid VM Allocation Algorithm
}

\author{
Inderjit Singh Dhanoa \\ Desh Bhagat University \\ Mandi Gobindgarh, \\ Punjab
}

\author{
Sawtantar Singh Khurmi, PhD \\ Desh Bhagat University \\ Mandi Gobindgarh, \\ Punjab
}

\begin{abstract}
Virtualization technology in Cloud computing has become important technology to reduce power consumption in data centers. Virtual Machine allocation to hosts is the main concept which carried out during Virtual Machine migrations in data centers. Virtual Machine allocation helps to utilize hardware resources of hosts and leads to power efficiency in Data centers. In the past few years, various mechanisms were proposed to apply algorithms to achieve power efficiency. In this paper, we have proposed a genetic algorithm to optimize various parameters i.e. power consumption, response time, SLA violation and VM migrations. Our proposed hybrid algorithm provisions various VMs to hosts in a way that to minimize power consumption, while delivering approved Quality of Service. Results demonstrate that proposed HVMA algorithm helps to minimize power consumption and to optimize various performance parameters during live migrations in various environment conditions.
\end{abstract}

\section{Keywords}

Data center, Virtualization, VM Allocation, Power Consumption, Virtual Machines (VMs), HVMA

\section{INTRODUCTION}

Cloud computing is gaining importance day-by-day. The large number enterprises and individuals are shifting and opting for cloud computing services. Thousands of servers have been employed worldwide to cater the needs of customers for computing services by big organizations like Amazon, Microsoft, IBM and Google. The round-the-clock reliable computational services, fault tolerance and information security are the main issues to be addressed while providing services to geographically spread customer sites [1]. Cloud computing, also known as "pay as you-go" utility model is economy driven. It becomes necessary for the service provider to ensure load balance and reliable computing services to its clients round the clock worldwide and keeping services ON means consuming power all the time to use resources [2].

To reduce power consumption of Data centers is an important issue because of large amount of electricity consumption. Mekinsey \& Company a consulting firm analyzed and stated that on an average only 6 to 8 percentage of total datacenter electricity power is used by their servers to perform computations [3].Thus; it is desirable to minimize power consumption in Data centers to reduce overall cost.

Virtualization technology helps to consolidate multiple VMs to lesser number of hosts and improves utilization of resources to reduce power consumption. VM consolidation can provide significant benefits to cloud computing by facilitating better use of the available data Center resources. Server consolidation using virtualization technology has become an important technology for improving the energy efficiency of data centers [4]. The basic idea behind the server consolidation technology is to perform migration of Virtual Machines (VMs) to as few energy efficient physical machines (PMs) as possible, and then switch off all the other PMs. The underlying computational problem of the server consolidation is basically a VM selection and placement problem, which has been elaborated in previous study [10]. In the past few years, many approaches to the VM consolidation problem have been proposed [5-9]. However, existing VM consolidation approaches do not consider the power consumption in the multi objective optimization scenario of parameters i.e. power consumption, response time, SLA violations and VM migrations of Data centers. In recent research studies, we have reviewed various papers [10] and analyzed the impact of VM size, migration time and network bandwidth parameters on energy consumption of subsystems [11].

A. Beloglazov et al. [9] presented the Modified Best-Fit Decreasing (MBFD) algorithm, which is best-fit decreasing heuristic, for Power-aware VM allocation and adaptive threshold-based migration algorithms to dynamic consolidation of VM resource partitions.

So, reducing power consumption is important and designing power-efficient data centers has recently received considerable attention of research community. Power consumption in data centers consists of two parts, including power consumed by the ICT (Information and Communications Technology) systems i.e. servers, storage and networking, and power consumed by infrastructure i.e. heating, ventilation and Air-Conditioning.

In this paper we have presented multi objective optimization of Modified Best Fit Decreasing Algorithm (MBFD) [9] in the light of various parameters like response time, SLA Violations, Power Consumption and VM migrations.

The remaining paper is organized as follows: section 2 discussing some literature review related with consolidation algorithms. Section 3 presents performance parameters to analyze optimization methodology of proposed algorithm. Section 4 discussing power consumption model to understand behavior of parameters. Section 5 describes Hybrid VM Allocation Algorithm in multi objective scenario. Section 6 presents evaluated performance and efficiency of HVMA algorithm in comparison with baseline algorithm on the basis of defined parameters. Section 7 concludes the paper with some future research work.

\section{LITERATURE REVIEW}

In the past few years, many approaches to the VM consolidation problem have been proposed [5-9].However; exiting VM placement methods do not consider multi objective parameter optimization during migrations using genetic Algorithms. Maolin Tang et al. [12] proposed a genetic algorithm for new VM placement problem that considers the energy consumption in both the servers and communication networks in the data centers as preliminary 
research. Further this study was extended to improve the performance and efficiency of genetic algorithm. Heena Kaushar et al. [13] aimed to analyze various VM consolidation algorithms based on various heuristics on legitimate host. Authors also presented a comparative study of various existing energy efficient VM consolidation algorithms using real world workload traces from more than a one thousand VMs using CloudSim toolkit. Anton Beloglazov et al. [14] presented a survey of research in energy-efficient computing. The architectural principles for energy-efficient management of Clouds, energy-efficient resource allocation policies and scheduling algorithms considering QoS expectations, power usage characteristics of the devices, and a number of open research challenges are addressed. This work substantially contributes to both resource providers and consumers. The approach is validated by conducting a performance evaluation study using the CloudSim $[15,16]$ toolkit showing significant cost savings and demonstrates high potential for the improvement of energy efficiency under dynamic workload scenarios. Bandi Madhusudhan et al. [17] designed a genetic algorithm which uses previous history and current demand of Virtual Machines in Placement decisions. Mohen Sharifi et al. [18] proposed an algorithm to schedule the workload of a set of virtual machines (VMs) to a set of physical machines (PMs) in a datacenter. The goal was to minimize total energy consumption by considering the conflicts between processor and disk utilizations and the costs of migrating VMs. To identify the conflicts, authors presented four models, namely the target system model, the application model, the energy model, and the migration model. Simulative results of proposed algorithm showed 24.9\% power savings compared to other methods. Fabio Lopez Pirez et al. [19] presented an extensive up- to-date most relevant VM consolidation literature review in order to identify research directions.

In this paper, we have proposed Hybrid VM Allocation Algorithm to optimize various parameters related with power consumption of underlying systems during VM Live Migrations.

\section{PERFORMANCE PARAMETERS}

To compare the efficiency of algorithms we used following various parameters to record their performance:

\subsection{Total Power Consumption}

Total power consumption is defined as the sum of power consumed by the physical resources of a data center as a result of application workloads and migration overhead.

\subsection{SLA Violations}

When a VM cannot get the promised Quality of Service (QoS), SLA violation takes place. For example when a VM cannot get requested MIPS (Million Instructions per Second), Then issue of SLA violation occurs.

\subsection{Response Time}

First response generated by Virtual Machine to client after submission of request to provide Quality of Service.

\subsection{Number of VM Migrations}

For dynamic VM consolidation it is must to determine overloaded and under-loaded hosts and once the overloaded or under-loaded hosts found the VMs get selected for migration. The minimization of the VM migration time is more important constraint and one of the ways to achieve it is to reduce the total number of VM migrations.

\section{POWER CONSUMPTION MODEL}

In Data centers, Mostly power is consumed by CPU, hard disk and network Interfaces. Out of these, CPU consumes major portion of energy in Data centers because of inefficient use of recourses and CPU utilization is directly proportional to workload. On average an idle server consumes approximately $70 \%$ of the power consumed by the server running at the full CPU speed [9].These studies justified that switching off idle servers to minimize power consumption is the efficient technique. Therefore, power model is defined as follows:

$$
P=k \cdot P_{\max }+(1-k) \cdot P_{\max } \cdot u
$$

$P_{\max }$ is the maximum power consumed when the server is fully utilized, $\mathrm{k}$ is the fraction of power consumed by idle server and $u$ is the CPU utilization.

Power consumption changes with time due to workload changes. As analysis study discussed in [11], energy consumption of underlying physical servers can be defined as follows:

$$
E_{\text {consumed }}=\int_{0}^{\mathrm{t}}\left(P_{t}-P_{0}\right) \mathrm{dt}
$$

\section{HYBRID VM ALLOCATION ALGORITHM}

Hybrid VM Allocation (HVMA) Algorithm is a method to optimize power consumption and performance in terms of response time, SLA violations and Number of VM migrations. Hybrid VM Allocation Algorithm is given as follows:

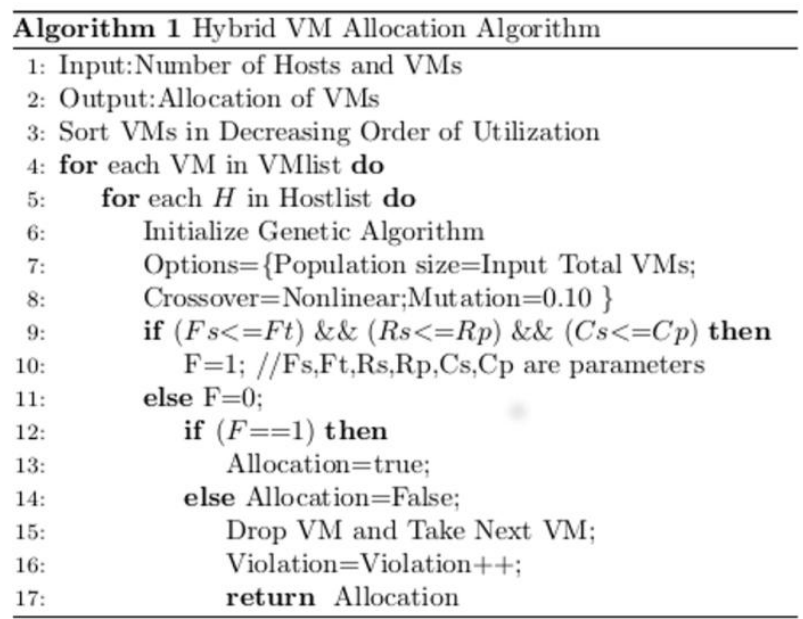

First initialize the VMs and define its parameters to describe the performance of VM Live migration for power consumption. At the same time initialize hosts to accept VMs after migration to accommodate the extra workload. Virtual Machines are sorted in decreasing order as per their utilization to provide services. One Allocation table of VMs is generated against each defined host. Then for each VM and host in VM allocation table initialize Genetic Algorithm [20] to improve the allocations and remove faulty VMs for proper utilizations. VMs with optimum threshold value or who are producing optimum value for all the defined parameters are accepted and placed on corresponding hosts as per allocation table.

Then fitness function is applied with various defined parameters and allocation of VMs accepted/rejected on basis of threshold value of target host. 


\section{PERFORMANCE EVALUATION}

The proposed HVMA has been validated with its implementation using MATLAB [21]. Since there are no benchmarks available for the new VM allocation problem, we have to randomly generate test problems to test the HVMA algorithm. Set of experiments were planned to evaluate the proposed HVMA algorithm with respect to performance parameters i.e. Power Consumption, VM Migrations, Response time and SLA Violations .In all the experiments, the population size of the HVMA algorithm varied from 100 to 500 for VMs, 5 to 20 for hosts, the probabilities for crossover and mutation were non linear and 0.10 , respectively, and the termination condition was 'no improvement' in the best solution for 10 generations. The various parameters like power consumption, VM migrations, SLA violations and response time were measured in KWatt, Number, Count and milliseconds respectively. Considering the stochastic nature of the Genetic Algorithm, experiments were repeated 10 times and recorded the solutions and computation times. Then mean values for all parameters were computed to draw the expected results in comparison with Base Algorithm (BA).

We evaluated the performance of the HVMA Algorithm by comparing the quality of the solutions generated by the HVMA Algorithm with the Quality of the solutions produced by the Base Algorithm (BA) which is based on heuristics defined by [9].

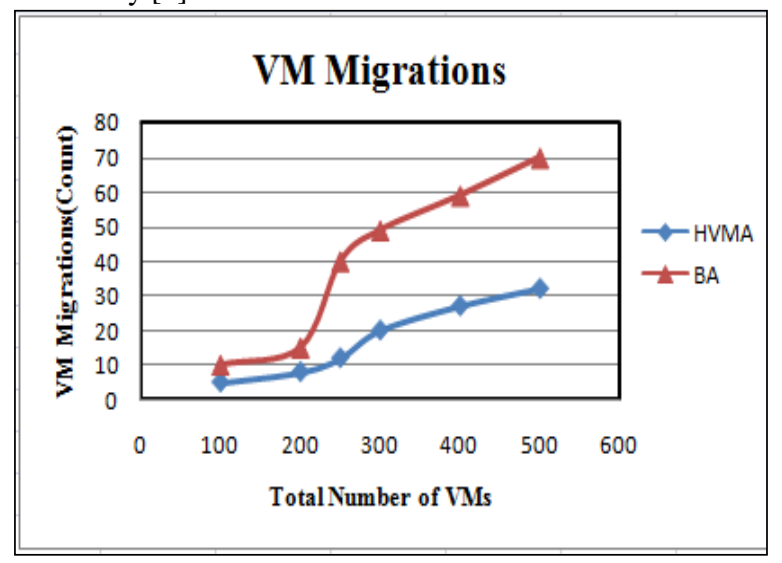

Fig 1: Number of VM Migrations

Fig.1 shows the comparison of HVMA algorithm and Base algorithm(BA) for VM Migration parameter.Comparison of values have shown the improvement in VM migrations with defined proposed Hybrid Genetic Algorithm i.e. decrease in number of VM migratuions.

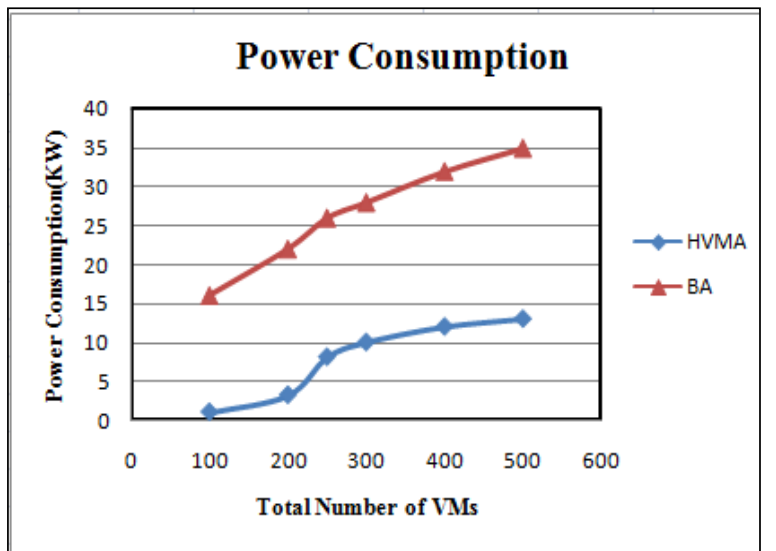

Fig 2: Comparison of Power consumption in $\mathrm{KW}$ for the respective migrated VMs
This Further reduces the live migration overhead of VMs from one host to another host from energy perspectve[22].

Fig.2 shows a comparison between Power consumption of Base Algorithm (BA) and proposed Genetic Algorithm with respect to various numbers of Virtual Machines in KW for the respective migrated VMs. For the same amount of migrated VMs, the power consumption of the base algorithm has been found to be more than that of the proposed Genetic algorithm. As per study [23], decrease in Virtual Machine Migrations leads to reduction in power consumption of underlying systems.

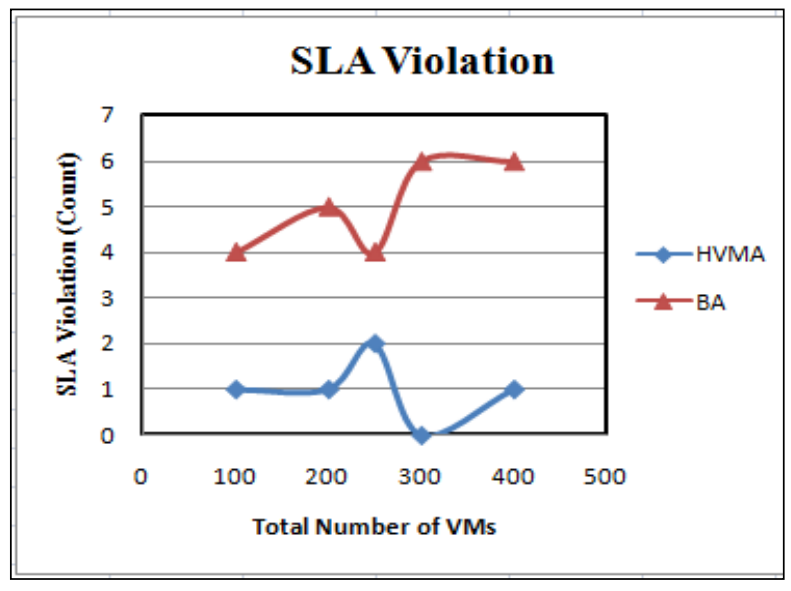

Fig 3: SLA violation comparison of HVMA and Base $\operatorname{algorithm(BA)}$

One of the important factors in this proposed algorithm is the SLA violation, whose comparative analysis with the base algorithm (BA) has been presented in Fig.3. The reason for the SLA Violation has been explained in the upper sections.SLA violation decreased with proposed Hybrid Genetic Algorithm as compare to earlier algorithm with the variation of Number Virtual Machines.

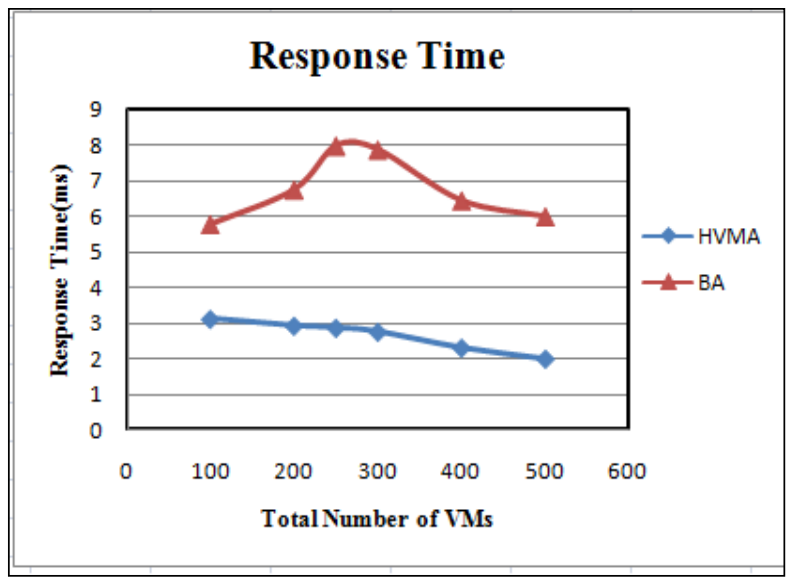

Fig 4: Response Time comparison of HVMA and Base $\operatorname{algorithm(BA)}$

Fig.4 shows the comparison of proposed and base algrithm(BA) in response time measured in milliseconds. Response time is the time interval taken by the system to respond for a particular event till the change of the state. Above figure shows the reduction in response time using HVMA algorithm with increase in number of Virtual Machines during the time. Quality of service provided by Virtual Machines can be maintained with decrease in value of 
Response time parameter. Congestion in network traffic during VM migrations is responsible for increase in response time of cloud data centers [24].

Table 1. HVMA Performance Optimization Trends (\%)

\begin{tabular}{|c|c|c|c|c|}
\hline $\begin{array}{c}\text { No. } \\
\text { of } \\
\text { VMs }\end{array}$ & $\begin{array}{l}\text { VM } \\
\text { Migrations }\end{array}$ & $\begin{array}{l}\text { Power } \\
\text { Consumption }\end{array}$ & $\begin{array}{l}\text { SLA } \\
\text { Violations }\end{array}$ & $\begin{array}{l}\text { Response } \\
\text { Time }\end{array}$ \\
\hline 100 & 50 & 93 & 75 & 46 \\
\hline 200 & 46 & 85 & 80 & 56 \\
\hline 250 & 70 & 68 & 50 & 64 \\
\hline 300 & 59 & 64 & 98 & 64 \\
\hline 400 & 54 & 62 & 83 & 64 \\
\hline 500 & 54 & 62 & 78 & 66 \\
\hline
\end{tabular}

It can be seen from the experimental results and trends shown in Table 1 that the solutions produced by the HVMA algorithm are significantly better than those produced by the Base algorithm. On average the solutions produced by the HVMA algorithm are $66 \%$ better than those Produced by the Base Algorithm (BA). In terms of power consumption, proposed HVMA algorithm is saving $72 \%$ of power as compare to base Algorithm with specified Quality of Service Constraints. Percentage of performance Trends have shown that various parameters are leading towards optimizations with great impact of HVMA Algorithm.

\section{CONCLUSION}

This paper has presented HVMA Algorithm for the allocation of Virtual Machines to hosts to optimize various performance parameters i.e. Power Consumption, VM Migrations, SLA Violations and Response Time. A new fitness function of the genetic algorithm in HVMA Algorithm has been applied and the performance compared with the defined base algorithm. The results of the HVMA algorithm have been found to be good enough in comparison to the base algorithm in terms of performance and efficiency. On average the solutions produced by the HVMA algorithm are $66 \%$ better than those produced by the Base Algorithm.

In future research, we will extend HVMA algorithm to optimize some more performance parameters during VM Live migrations with different scenario and within defined Quality of Service constraints.

\section{REFERENCES}

[1] Buyya, R., Yeo, C. S., Venugopal, S., Broberg, J., \& Brandic, I. (2009). Cloud computing and emerging IT platforms: Vision, hype, and reality for delivering computing as the $5^{\text {th }}$ utility. Future Generation computer systems, 25(6), 599-616.

[2] Pedram, M. (2012). Energy-efficient data centers. Computer-Aided Design of Integrated Circuits and Systems, IEEE Transactions on, 31(10), 1465-1484.

[3] Glanz, J. (2012). Power, pollution and the internet. The New York Times, 22.

[4] Meng, X., Pappas, V., \& Zhang, L. (2010, March). Improving the scalability of data center networks with traffic-aware virtual machine placement. In INFOCOM, 2010 Proceedings IEEE (pp. 1-9).
[5] Verma, A., Ahuja, P., \& Neogi, A. (2008). pMapper: power and migration cost aware application placement in virtualized systems. In Middleware 2008 (pp. 243-264). Springer Berlin Heidelberg.

[6] Hermenier, F., Lorca, X., Menaud, J. M., Muller, G., \& Lawall, J. (2009, March). Entropy: a consolidation manager for clusters. In Proceedings of the 2009 ACM SIGPLAN/SIGOPS International conference on Virtual execution environments (pp. 41-50).

[7] Xu, J., \& Fortes, J. A. (2010, December). Multi-objective virtual machine placement in virtualized data center environments. In Green Computing and Communications (GreenCom), 2010 IEEE/ACM Int'l Conference on Cyber, Physical and Social Computing (CPSCom) (pp. 179-188).

[8] Stillwell, M., Schanzenbach, D., Vivien, F., \& Casanova, H. (2010). Resource allocation algorithms for virtualized service hosting platforms. Journal of Parallel and distributed Computing, 70(9), 962-974.

[9] Beloglazov, A., Abawajy, J., \& Buyya, R. (2012). Energy-aware resource allocation heuristics for efficient management of data centers for cloud computing. Future generation computer systems, 28(5), 755-768.

[10] Dhanoa, I. S., \& Khurmi, D. S. S. (2014). Energyefficient virtual machine live migration in cloud data centers. International Journal of Computer Science and Technology (IJCST), 5(1), 43-47.

[11] Dhanoa, I. S., \& Khurmi, S. S. (2015). Analyzing Energy Consumption during VM Live Migration. Published in IEEE International Conference on Computing, Communication and Automation (ICCCA2015), (pp.584588).

[12] Tang, M., \& Pan, S. (2014). A hybrid genetic algorithm for the energy-efficient virtual machine placement problem in data centers. Neural Processing Letters, 41(2), 211-221.

[13] Kaushar, H., Ricchariya, P., \& Motwani, A. (2014). Comparison of SLA based Energy Efficient Dynamic Virtual Machine Consolidation Algorithms. International Journal of Computer Applications, 102(16).

[14] Buyya, R., Beloglazov, A., \& Abawajy, J. (2010). Energy-efficient management of data center resources for cloud computing: a vision, architectural elements, and open challenges. arXiv preprint arXiv:1006.0308.

[15] Beloglazov, A., \& Buyya, R. (2012). Optimal online deterministic algorithms and adaptive heuristics for energy and performance efficient dynamic consolidation of virtual machines in cloud data centers. Concurrency and Computation: Practice and Experience, 24(13), 1397-1420.

[16] Beloglazov, A., \& Buyya, R. (2010, May). Energy efficient allocation of virtual machines in cloud data centers. In Cluster, Cloud and Grid Computing (CCGrid), $201010^{\text {th }}$ IEEE/ACM International Conference on (pp. 577-578).

[17] Madhusudan, B. \& Sekaran, K. (2013). A Genetic Algorithm Approach for Virtual Machine Placement in Cloud. Published in the proceeding of International 
Conference on Emerging Research in Computing, Information, Communication and Applications (ERCICA2013).

[18] Sharifi, M., Salimi, H., \& Najafzadeh, M. (2012). Powerefficient distributed scheduling of virtual machines using workload-aware consolidation techniques. The Journal of Supercomputing, 61(1), 46-66.

[19] Lopez-Pires, F., \& Baran, B. (2015). Virtual Machine Placement Literature Review. arXiv preprint arXiv:1506.01509.

[20] Popov, A. (2005). Genetic algorithms for optimization. User Manual, Hamburg, 2013.

[21] Grace, A. (1990). Optimization Toolbox: For Use with MATLAB: User's Guide. M. Works (Ed.). Math works.
[22] Strunk, A., \& Dargie, W. (2013, March). Does live migration of virtual machines cost energy?. In Advanced Information Networking and Applications (AINA), 2013 IEEE 27th International Conference on (pp. 514-521).

[23] Rybina, K., Dargie, W., Strunk, A., \& Schill, A. (2013, October). Investigation into the energy cost of live migration of virtual machines. In Sustainable Internet and ICT for Sustainability (SustainIT), 2013 (pp. 1-8).

[24] Kikuchi, S., \& Matsumoto, Y. (2012, June). Impact of live migration on multi-tier application performance in clouds. In Cloud Computing (CLOUD), 2012 IEEE 5th International Conference on (pp. 261-268). 